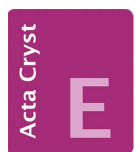

CRYSTALLOGRAPHIC COMMUNICATIONS

ISSN 2056-9890

Received 10 November 2017

Accepted 16 November 2017

Edited by D. Chopra, Indian Institute of Science Education and Research Bhopal, India

Keywords: crystal structure; hydrogen bonding; barbiturates; pharmaceuticals.

CCDC references: $1586016 ; 1586015$

Supporting information: this article has supporting information at journals.iucr.org/e

\title{
Buthalital and methitural - 5,5-substituted derivatives of 2-thiobarbituric acid forming the same type of hydrogen-bonded chain
}

\author{
Thomas Gelbrich* and Ulrich J. Griesser
}

University of Innsbruck, Institute of Pharmacy, Innrain 52, 6020 Innsbruck, Austria. *Correspondence e-mail:
thomas.gelbrich@uibk.ac.at

The molecule of buthalital, (I) [systematic name: 5-(2-methylpropyl)-5-(prop-2en-1-yl)-2-sulfanylidene-1,3-diazinane-4,6-dione], $\mathrm{C}_{11} \mathrm{H}_{16} \mathrm{~N}_{2} \mathrm{O}_{2} \mathrm{~S}$, exhibits a planar pyrimidine ring, whereas the pyrimidine ring of methitural, (II) [systematic name: 5-(1-methylbutyl)-5-[2-(methylsulfanyl)ethyl]-2-sulfanylidene-1,3-diazinane-4,6-dione], $\mathrm{C}_{12} \mathrm{H}_{20} \mathrm{~N}_{2} \mathrm{O}_{2} \mathrm{~S}_{2}$, is slightly puckered. (I) and (II) contain the same hydrogen-bonded chain structure in which each molecule is connected, via four $\mathrm{N}-\mathrm{H} \cdots \mathrm{O}=\mathrm{C}$ hydrogen bonds, to two other molecules, resulting in a hydrogen-bonded chain displaying a sequence of $R_{2}^{2}(8)$ rings. The same type of $\mathrm{N}-\mathrm{H} \cdots \mathrm{O}=\mathrm{C}$ hydrogen-bonded chain has previously been found in several 5,5-disubstituted derivatives of barbituric acid which are chemically closely related to (I) and (II).

\section{Chemical context}

Buthalital (I) and methitural (II) are 5,5-disubstituted derivatives of 2-thiobarbituric acid. Compounds of the thiobarbiturate class differ from the corresponding barbiturates in that the ketone group at the 2-position is replaced by a thione group. Thiobarbiturates are used as injection narcotics for the induction of general anaesthesia or to produce complete anaesthesia of short duration. The sodium salt of (I) was originally developed as a short-acting anaesthetic but was found to have an extremely rapid elimination rate. Similarly, (II) was marketed in the 1950 s as an ultra-short-acting intravenous anaesthetic.

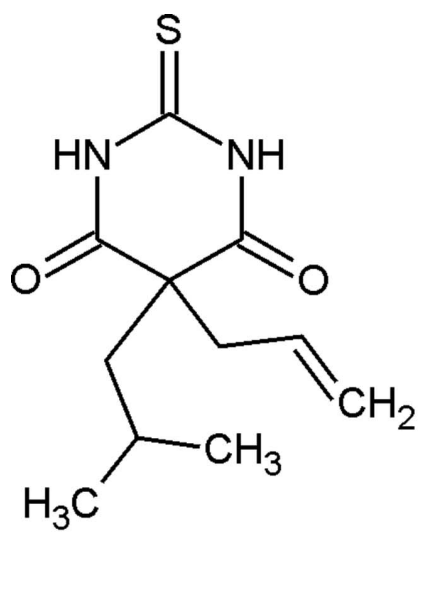

(I)<smiles>CCCC(C)C1(CCSC)C(=O)NC(=S)NC1=O</smiles>

(II) 


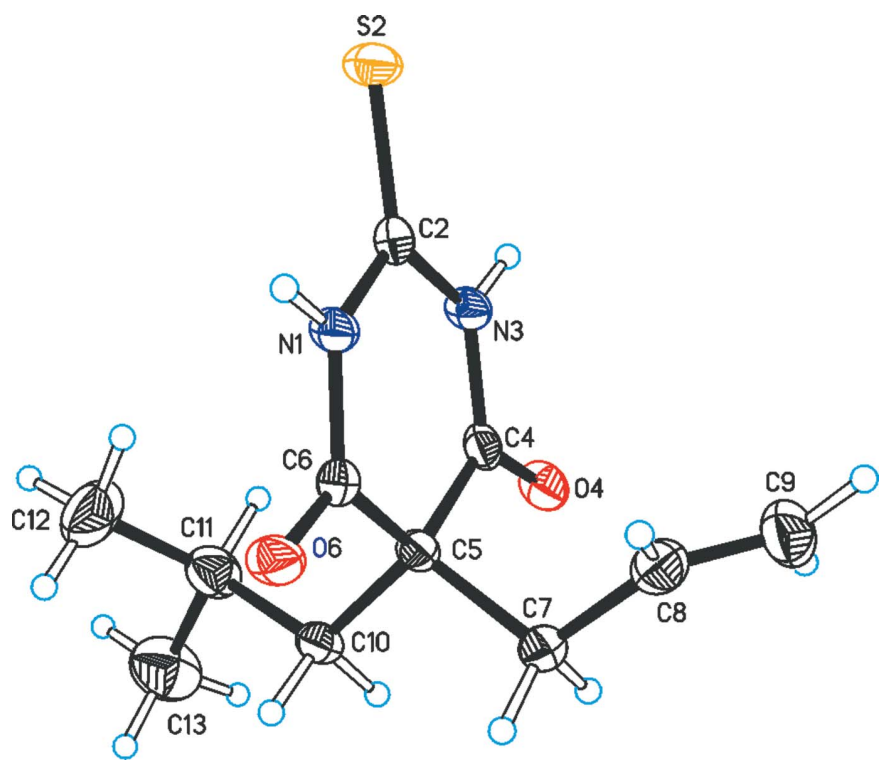

Figure 1

The molecular structure of (I), with displacement ellipsoids drawn at the $50 \%$ probability level and $\mathrm{H}$ atoms drawn as spheres of arbitrary size.

\section{Structural commentary}

The molecular structure of (I), Fig. 1, shows an almost planar pyrimidine ring $(\mathrm{N} 1, \mathrm{C} 2, \mathrm{~N} 3, \mathrm{C} 4 \mathrm{C} 5, \mathrm{C} 6)$ with a root-meansquare (r.m.s.) deviation of its six atoms from the mean plane of $0.016 \AA$ (Fig. 1). The (C7, C8, C5, C10, C11) unit defined by ring atom $\mathrm{C} 5$ and two atoms of each of the allyl and isobutyl substituents is nearly planar (r.m.s. deviation $=0.050 \AA$ ). The

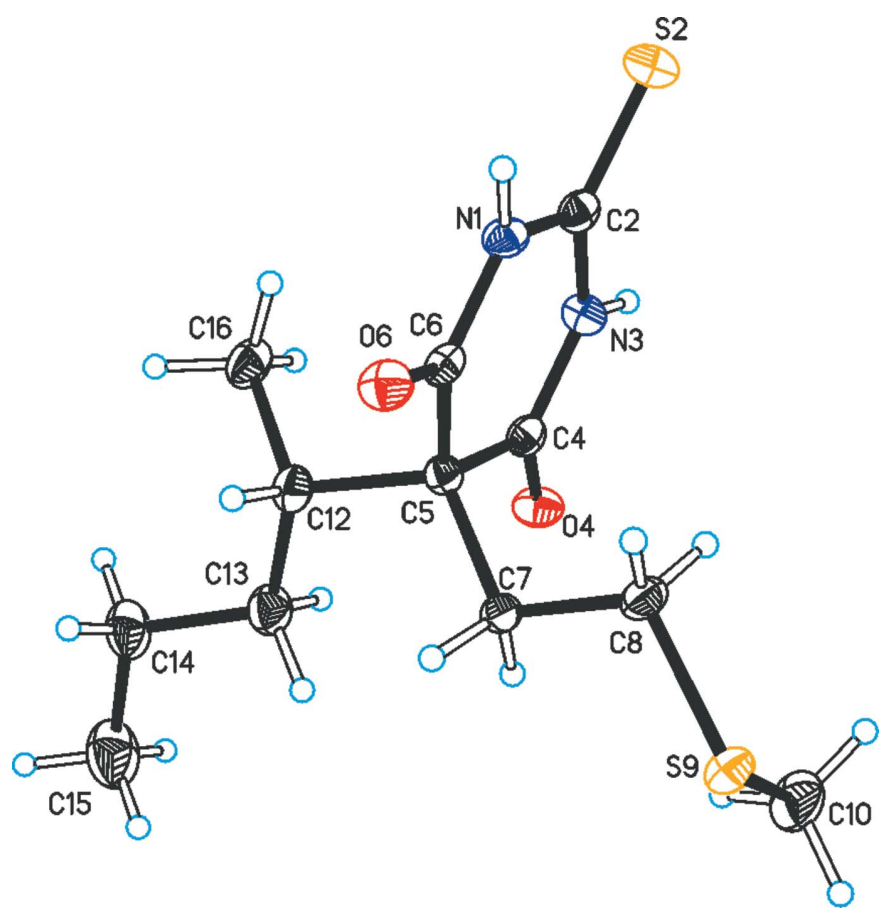

Figure 2

The molecular structure of (II), with displacement ellipsoids drawn at the $50 \%$ probability level and $\mathrm{H}$ atoms drawn as spheres of arbitrary size.
Table 1

Hydrogen-bond geometry $\left(\AA,^{\circ}\right)$ for $(\mathrm{I})$.

\begin{tabular}{lllll}
\hline$D-\mathrm{H} \cdots A$ & $D-\mathrm{H}$ & $\mathrm{H} \cdots A$ & $D \cdots A$ & $D-\mathrm{H} \cdots A$ \\
\hline $\mathrm{N} 1-\mathrm{H} 1 \cdots \mathrm{O}^{\mathrm{i}}$ & $0.87(2)$ & $1.95(2)$ & $2.815(2)$ & $174(2)$ \\
$\mathrm{N} 3-\mathrm{H} 3 \cdots 6^{\mathrm{ii}}$ & $0.86(2)$ & $2.10(2)$ & $2.922(2)$ & $160(2)$ \\
\hline
\end{tabular}

Symmetry codes: (i) $-x+\frac{1}{2}, y+\frac{1}{2},-z+\frac{3}{2}$; (ii) $-x+\frac{1}{2}, y-\frac{1}{2},-z+\frac{3}{2}$.

mean plane of this fragment forms an angle of $87.5(1)^{\circ}$ with the plane of the six-membered ring. Additionally, it forms an angle of $77.8(2)^{\circ}$ with the plane of the allyl group defined by $\mathrm{C} 7, \mathrm{C} 8$ and $\mathrm{C} 9$. The terminal torsion angles $\mathrm{C} 5-\mathrm{C} 10-\mathrm{C} 11-$ $\mathrm{C} 12$ and $\mathrm{C} 5-\mathrm{C} 10-\mathrm{C} 11-\mathrm{C} 13$ of the isobutyl substituent are $-71.7(3)^{\circ}$ and $165.6(2)^{\circ}$, respectively.

The pyrimidine ring (N1, C2, N3, C4 C5, C6) in the molecule of (II) deviates somewhat from planarity (r.m.s. deviation $=0.030 \AA$ ); specifically, the distance between C6 and the mean plane defined by the other five ring atoms (r.m.s deviation $=0.005 \AA$ ) is 0.104 (2) $\AA$ (Fig. 2). The mean plane of the ( $\mathrm{S} 9, \mathrm{C} 8, \mathrm{C} 7, \mathrm{C} 5, \mathrm{C} 12, \mathrm{C} 16)$ chain, defined by ring atom $\mathrm{C} 6$, three atoms of the 2-(methylthio)ethyl substituent and two atoms of the sec-butyl group (r.m.s. deviation $=0.091 \AA$ ) forms an angle of $88.64(5)^{\circ}$ with the mean plane of the pyrimidine ring and an angle of $39.0(1)^{\circ}$ with the mean plane of the (C5, $\mathrm{C} 12, \mathrm{C} 13, \mathrm{C} 14, \mathrm{C} 15)$ fragment of the nearly planar (r.m.s. deviation $=0.070$ ) sec-butyl group. In the 2-(methylthio)ethyl substituent, the $\mathrm{C} 10-\mathrm{S} 9$ and $\mathrm{C} 8-\mathrm{S} 9$ bond lengths are 1.794 (2) and 1.803 (2) $\AA$, respectively, and the C7-C8-S9$\mathrm{C} 10$ torsion angle is $82.5(2)^{\circ}$. The bond between ring atom $\mathrm{C} 5$ and atom C12 of the sec-butyl group [1.582 (2) $\AA$ ] is somewhat longer than the analogous distance between $\mathrm{C} 5$ and atom $\mathrm{C} 7$ of the 2-(methylthio)ethyl group [1.547 (2) ̊̊]. This difference is reminiscent of the difference between equatorial and axial bonds at ring atom C5 found in several 5,5-disubstituted barbituric acid derivatives that exhibit a puckered pyrimidine ring (Gelbrich et al., 2016b).

\section{Supramolecular features}

The crystal structure of (I) contains $\mathrm{N} 1-\mathrm{H} 1 \cdots \mathrm{O} 4^{\mathrm{i}}$ and $\mathrm{N} 3-$

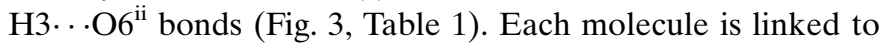
two neighbouring molecules via two-point connections and $R_{2}^{2}(8)$ rings (Etter et al. 1990, Bernstein et al., 1995). The resulting chain structure (topological type 2C1) contains a twofold screw axis and runs parallel to the $b$ axis. The mean planes of neighbouring pyrimidine rings in the chain form an angle of approximately $40^{\circ}$ with one another. The chain structure of (I) belongs to the $\mathbf{C - 2}$ type, which also occurs in a number of 5,5-disubstituted barbituric acid derivatives (Gelbrich et al., 2016a). The four shortest intermolecular contacts of the sulfur atom (S. . H distances between 2.97 and $3.01 \AA$; close to the sum of van der Waals radii) involve both $\mathrm{CH}_{2}$ groups of a neighbouring molecule and one $\mathrm{CH}_{3}$ group belonging to the isobutyl substituent of two other molecules.

Two independent hydrogen bonds, $\mathrm{N} 1-\mathrm{H} 1 \cdots \mathrm{O} 6^{\mathrm{i}}$ and $\mathrm{N} 3-$ $\mathrm{H} 3 \cdots \mathrm{O} 4^{\mathrm{ii}}$, are present in the crystal structure of (II). As in (I), each molecule is linked, by two-point connections, to two 
Table 2

Hydrogen-bond geometry $\left(\AA,^{\circ}\right)$ for (II).

\begin{tabular}{lllll}
\hline$D-\mathrm{H} \cdots A$ & $D-\mathrm{H}$ & $\mathrm{H} \cdots A$ & $D \cdots A$ & $D-\mathrm{H} \cdots A$ \\
\hline $\mathrm{N} 1-\mathrm{H} 1 \cdots \mathrm{O} 6^{\mathrm{i}}$ & $0.86(2)$ & $2.07(2)$ & $2.921(2)$ & $170(2)$ \\
$\mathrm{N} 3-\mathrm{H} 3 \cdots \mathrm{O} 4^{\mathrm{ii}}$ & $0.86(2)$ & $2.14(2)$ & $2.963(2)$ & $160(2)$ \\
\hline
\end{tabular}

Symmetry codes: (i) $-x,-y+1,-z+1$; (ii) $-x, y,-z+\frac{1}{2}$.

neighbouring molecules so that a $\mathbf{C - 2}$ chain structure is formed that propagates parallel to the $c$ axis. In this case, the $\mathbf{C - 2}$ chain contains two crystallographically distinct $R_{2}^{2}(8)$ rings which are centred either by a twofold axis or an inversion centre (Fig. 4, Table 2). The mean planes of adjacent pyrimidine rings in the same chain are either coplanar with one another (if the corresponding molecules are related by an inversion operation), or they form an angle of $75^{\circ}$ (if the molecules are related by a $180^{\circ}$ rotation). The sulfur atom S9 of the 2-(methylthio)ethyl substituent forms an intermolecular contact $(\mathrm{S} \cdots \mathrm{H}$ $=2.86 \AA$ ) with the $s e c$-butyl group of a molecule belonging to a neighbouring chain and S2 lies in close proximity to the methyl group of a 2-(methylthio)ethyl substituent $(\mathrm{S} \cdots \mathrm{H}=$ $2.96 \AA$ ).

\section{Database survey}

The crystal structures of three polymorphs of the keto form of 2-thiobarbituric acid, which is a close structural analogue of (I) and (II), have been determined (Chierotti et al., 2010). Polymorph III (CSD refcode THBARB01) contains an N-H..Obonded layer structure having the hcb topology and polymorph IV (THBARB02) an $\mathrm{N}-\mathrm{H} \cdots$ O-bonded framework. Both these structures contain $\mathrm{N}-\mathrm{H} \cdots \mathrm{O}$-bonded $R_{2}^{2}(8)$ rings

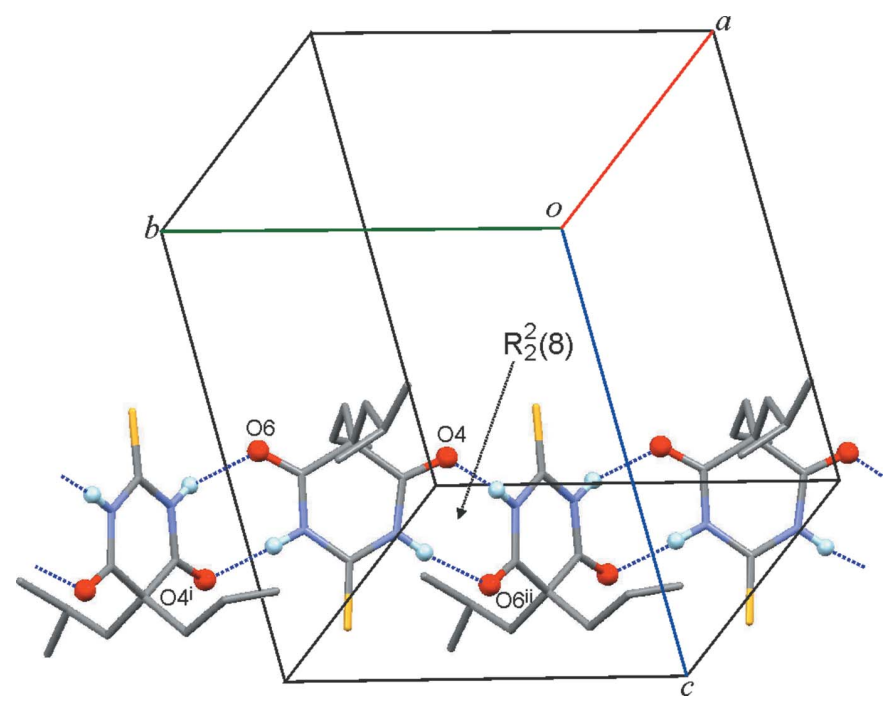

Figure 3

The C-2-type bonded chain of (I). $\mathrm{O}$ and $\mathrm{H}$ atoms directly involved in $\mathrm{N}-$ $\mathrm{H}$. . O interactions are drawn as balls and $\mathrm{H}$ atoms bonded to $\mathrm{C}$ atoms are omitted for clarity. The chain displays a twofold screw symmetry and contains just one type of $R_{2}^{2}(8)$ ring. [Symmetry codes: (i) $-x+\frac{1}{2}, y+\frac{1}{2}$, $-z+\frac{3}{2}$; (ii) $-x+\frac{1}{2}, y-\frac{1}{2},-z+\frac{3}{2}$.]

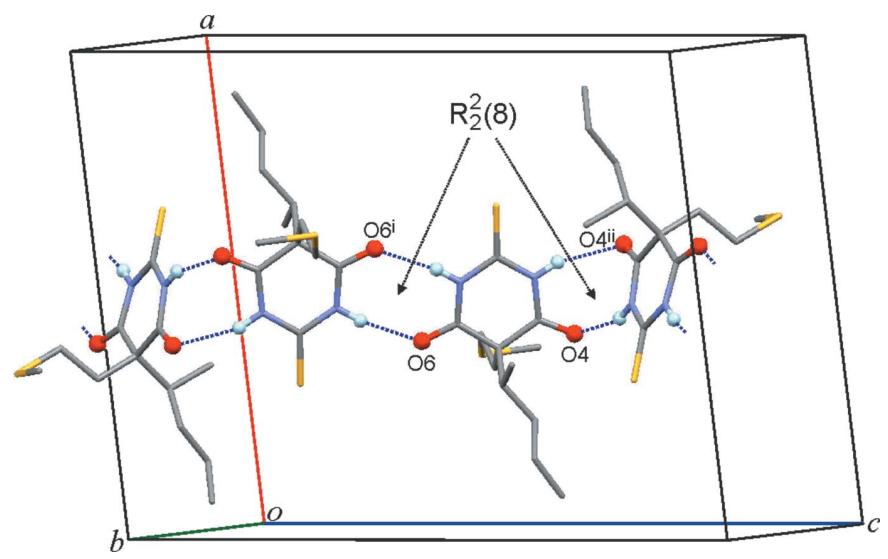

Figure 4

The C-2-type bonded chain of (II). $\mathrm{O}$ and $\mathrm{H}$ atoms directly involved in $\mathrm{N}-\mathrm{H} \cdots \mathrm{O}$ interactions are drawn as balls and $\mathrm{H}$ atoms bonded to $\mathrm{C}$ atoms are omitted for clarity. The chain displays two types of $R_{2}^{2}(8)$ ring, which contain an inversion centre $\left(\mathrm{N} 1-\mathrm{H} 1 \cdots \mathrm{O} 6^{\mathrm{i}}\right)$ or a twofold axis $\left(\mathrm{N} 3-\mathrm{H} 3 \cdots \mathrm{O}^{\mathrm{ii}}\right)$. [Symmetry codes: (i) $-x,-y+1,-z+1$; (ii) $-x, y$, $-z+\frac{1}{2}$.]

analogous to those present in the hydrogen-bonded chains of (I) and (II), and additionally they contain one-point hydrogen-bond connections between molecules. Form VI of 2-thiobarbituric acid (THBARB03) displays two distinct hydrogen-bonded structures, an $\mathrm{N}-\mathrm{H}$... O-bonded layer with sql topology whose molecules are linked exclusively by onepoint connections and an hcb-type layer based on $\mathrm{N}-\mathrm{H} \cdots \mathrm{O}$ (a)

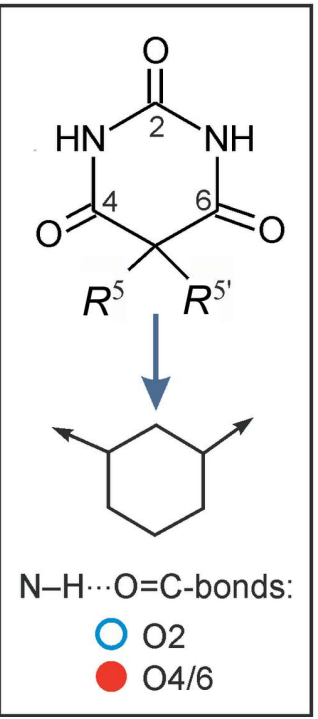

(b)

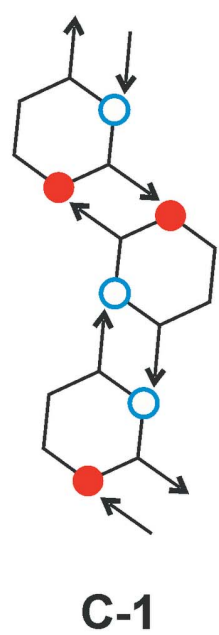

(c)

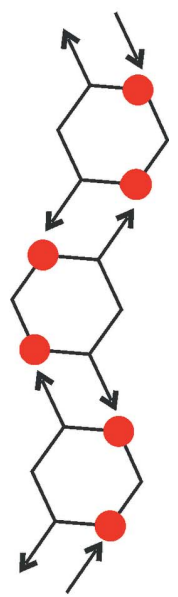

C-2
Figure 5

(a) Simplified representation of a molecule of a 5,5-disubstituted derivative of barbituric acid. The same scheme can be applied for analogous thiobarbiturates such as (I) and (II) if the $\mathrm{O}$ atom of the carbonyl group in position 2 is replaced by a thioxo $\mathrm{S}$ atom. $(b)$ and $(c)$ Schematic representation of the $\mathrm{N}-\mathrm{H} \cdots \mathrm{O}=\mathrm{C}$-bonded chain types $\mathbf{C}-\mathbf{1}$ and $\mathbf{C - 2}$ with an underlying $2 \mathrm{C} 1$ topology, which are frequently found in barbiturates. The thiobarbiturates (I) and (II) contain chains of the $\mathbf{C - 2}$ type. 
Table 3

Experimental details.

(I)

Crystal data

Chemical formula

$M_{\mathrm{r}}$

Crystal system, space group

Temperature (K)

$a, b, c(\AA)$

$\beta\left({ }^{\circ}\right)$

$V\left(\AA^{3}\right)$

Z

Radiation type

$\mu\left(\mathrm{mm}^{-1}\right)$

Crystal size (mm)

Data collection

Diffractometer

Absorption correction

$T_{\min }, T_{\max }$

No. of measured, independent and $R_{\text {int }}$ observed $[I>2 \sigma(I)]$ reflections

Refinement

$R\left[F^{2}>2 \sigma\left(F^{2}\right)\right], w R\left(F^{2}\right), S$

No. of reflections

No. of parameters

No. of restraints

$\mathrm{H}$-atom treatment

$\Delta \rho_{\max }, \Delta \rho_{\min }\left(\mathrm{e} \AA^{-3}\right)$

\author{
$\mathrm{C}_{11} \mathrm{H}_{16} \mathrm{~N}_{2} \mathrm{O}_{2} \mathrm{~S}$ \\ 240.32 \\ Monoclinic, $P 2_{1} / n$ \\ 120 \\ 8.7271 (6), 11.6521 (4), 12.5400 (8) \\ $96.539(2)$ \\ $1266.89(13)$ \\ 4 \\ Mo $K \alpha$ \\ 0.24 \\ $0.40 \times 0.10 \times 0.05$
}
Bruker-Nonius Roper CCD camera on $\kappa$ - goniostat
Multi-scan (SADABS; Sheldrick, 2007)
$0.924,1.000$
$9476,2519,1833$
0.067
$0.045,0.108,1.04$
2519
170
2
$\mathrm{H}$ atoms treated by a mixture of independent and constrained refinement
$0.31,-0.27$

(II)

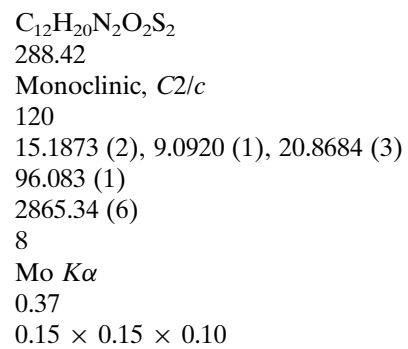

Bruker-Nonius APEXII CCD camera on $\kappa$ goniostat

Multi-scan (SADABS; Sheldrick, 2007)

$0.974,1.000$

$24772,2813,2630$

0.034

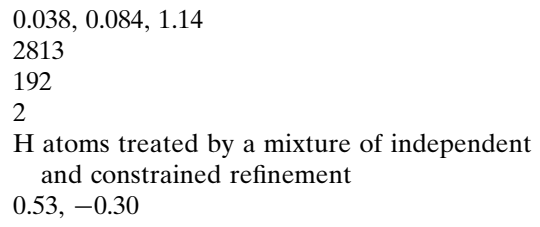

Computer programs: DENZO (Otwinowski \& Minor, 1997), COLLECT (Hooft, 1998), SHELXT (Sheldrick, 2015a), SHELXL2014 (Sheldrick, 2015b), XP in SHELXTL (Sheldrick, 2008) Mercury (Macrae et al., 2006), PLATON (Spek, 2009), publCIF (Westrip, 2010) and TOPOS (Blatov, 2006).

as well as $\mathrm{N}-\mathrm{H} \cdots \mathrm{S}$ bonds, with the latter interaction resulting in $R_{2}^{2}(8)$ rings.

Numerous 5,5-substituted derivatives of barbituric acid are known to form $\mathrm{N}-\mathrm{H} \cdots \mathrm{O}=\mathrm{C}$-bonded chains exhibiting the $2 \mathrm{C} 1$ topology, with their molecules being linked by two-point connections resulting in the formation of characteristic $R_{2}^{2}(8)$ rings. Chains exhibiting these specific properties can be classified into two distinct types, denoted as C-1 and C-2 (Gelbrich et al., 2016a; see Fig. 5). The less frequent of these two types, C-2, is also the chain motif of (I) and (II). It is characterized by the employment of each of the topologically equivalent $\mathrm{C} 4$ and C6 carbonyl groups, but not the $\mathrm{C} 2$ group, as a hydrogenbond acceptor.

C-2 chains containing a 2 screw axis occur in polymorph III of phenobarbital (PHBARB09), the $\mathrm{CH}_{2} \mathrm{Cl}_{2}$ solvate of the same compound (EPUDEA) (Zencirci et al., 2010, 2014) and in 5-fluoro-5-phenylbarbituric acid (HEKTOG) (DesMarteau et al., 1994) as well as in (I). By contrast, the C-2 chains of 6oxocyclobarbital (OXCBAR) (Chentli-Benchikha et al., 1977) and polymorph III of pentobarbital (FUFTEG02) (Rossi et al., 2012) exhibit glide symmetry. Moreover, polymorph II of barbital (DETBAA02) (Craven et al., 1969) as well as forms I and II of phenobarbital (Zencirci et al., 2010) exhibit C-2 chains whose $R_{2}^{2}(8)$ rings contain crystallographic inversion centres. The crystal structure of methitural (II) is the first example of a $\mathbf{C - 2}$ chain whose $R_{2}^{2}(8)$ rings are centred alternately by a twofold rotational axis and an inversion centre.

\section{Synthesis and crystallization}

Single crystals of (I) were produced by sublimation between two glass slides separated by a spacer ring (height: $1 \mathrm{~cm}$ ), using a hot bench at a temperature of $403 \mathrm{~K}$. As confirmed by PXRD, the phase investigated by us is identical with that of the original sample from the1940s obtained from our archive. The melting point of this phase of $422 \mathrm{~K}$ was determined with hot-stage microscopy. Heating the quench-cooled melt of (I) above $323 \mathrm{~K}$ resulted in the crystallization of a second form. Isolated, individual crystals of this second form melted at approximately $387 \mathrm{~K}$. In other experiments, a phase transition from the low-melting form II to a high-melting form I occurred on heating, usually between 378 and $383 \mathrm{~K}$ (see Supporting information). These observations are consistent with a previous description by Brandstätter-Kuhnert \& Aepkers (1962).

The crystals of (II) investigated in this study were taken from a sample obtained from Merck AG, Darmstadt, Germany. These crystals melted within a relatively broad temperature range between 361 and $366 \mathrm{~K}$.

\section{Refinement}

Crystal data, data collection and structure refinement details are summarized in Table 3. All $\mathrm{H}$ atoms were identified in difference maps. Methyl $\mathrm{H}$ atoms were idealized and included 
as rigid groups allowed to rotate but not tip and all other $\mathrm{H}$ atoms bonded to carbon atoms were positioned geometrically $(\mathrm{C}-\mathrm{H}=0.95-0.99 \AA)$. The hydrogen atoms in $\mathrm{NH}$ groups were refined with restrained distances $[\mathrm{N}-\mathrm{H}=0.88$ (2) $\AA$ ]. The $U_{\text {iso }}$ parameters of all $\mathrm{H}$ atoms were refined freely.

\section{Acknowledgements}

We thank Professor S. Coles (Southampton) for providing access to the X-ray diffractometers used in this study.

\section{References}

Bernstein, J., Davis, R. E., Shimoni, L. \& Chang, N.-L. (1995). Angew. Chem. Int. Ed. Engl. 34, 1555-1573.

Blatov, V. A. (2006). IUCr Compcomm Newsl. 7, 4-38.

Brandstätter-Kuhnert, M. \& Aepkers, M. (1962). Mikrochim. Acta, 50, 1055-1074.

Chentli-Benchikha, F., Declercq, J. P., Germain, G., Van Meerssche, M., Bouché, R. \& Draguet-Brughmans, M. (1977). Acta Cryst. B33, 2739-2743.

Chierotti, M. R., Ferrero, L., Garino, N., Gobetto, R., Pellegrino, L., Braga, D., Grepioni, F. \& Maini, L. (2010). Chem. Eur. J. 16, 43474358.

Craven, B. M., Vizzini, E. A. \& Rodrigues, M. M. (1969). Acta Cryst. B25, 1978-1993.

DesMarteau, D. D., Pennington, W. T. \& Resnati, G. (1994). Acta Cryst. C50, 1305-1308.
Etter, M. C., MacDonald, J. C. \& Bernstein, J. (1990). Acta Cryst. B46, 256-262.

Gelbrich, T., Braun, D. E. \& Griesser, U. J. (2016a). Chem. Cent. J. 10, 8.

Gelbrich, T., Braun, D. E., Oberparleiter, S., Schottenberger, H. \& Griesser, U. J. (2016b). Crystals, 6, 47.

Hooft, R. W. W. (1998). COLLECT. Nonius BV, Delft, The Netherlands.

Macrae, C. F., Edgington, P. R., McCabe, P., Pidcock, E., Shields, G. P., Taylor, R., Towler, M. \& van de Streek, J. (2006). J. Appl. Cryst. 39, 453-457.

Otwinowski, Z. \& Minor, W. (1997). Methods in Enzymology, Vol. 276, Macromolecular Crystallography, Part A, edited by C. W. Carter Jr \& R. M. Sweet, pp. 307-326. New York: Academic Press.

Rossi, D., Gelbrich, T., Kahlenberg, V. \& Griesser, U. J. (2012). CrystEngComm, 14, 2494-2506.

Sheldrick, G. M. (2007). SADABS. University of Göttingen, Germany.

Sheldrick, G. M. (2008). Acta Cryst. A64, 112-122.

Sheldrick, G. M. (2015a). Acta Cryst. A71, 3-8.

Sheldrick, G. M. (2015b). Acta Cryst. C71, 3-8.

Spek, A. L. (2009). Acta Cryst. D65, 148-155.

Westrip, S. P. (2010). J. Appl. Cryst. 43, 920-925.

Zencirci, N., Gelbrich, T., Apperley, D. C., Harris, R. K., Kahlenberg, V. \& Griesser, U. J. (2010). Cryst. Growth Des. 10, 302-313.

Zencirci, N., Griesser, U. J., Gelbrich, T., Kahlenberg, V., Jetti, R. K. R., Apperley, D. C. \& Harris, R. K. (2014). J. Phys. Chem. B, 118, 3267-3280. 


\section{supporting information}

Acta Cryst. (2017). E73, 1908-1912 [https://doi.org/10.1107/S205698901701653X]

\section{Buthalital and methitural - 5,5-substituted derivatives of 2-thiobarbituric acid forming the same type of hydrogen-bonded chain}

\section{Thomas Gelbrich and Ulrich J. Griesser}

\section{Computing details}

For both structures, data collection: COLLECT (Hooft, 1998); cell refinement: DENZO (Otwinowski \& Minor, 1997) and COLLECT (Hooft, 1998); data reduction: DENZO (Otwinowski \& Minor, 1997) and COLLECT (Hooft, 1998); program(s) used to solve structure: SHELXT (Sheldrick, 2015a); program(s) used to refine structure: SHELXL2014 (Sheldrick, 2015b); molecular graphics: XP in SHELXTL (Sheldrick, 2008) and Mercury (Macrae et al., 2006). Software used to prepare material for publication: PLATON (Spek, 2009), publCIF (Westrip, 2010) and TOPOS (Blatov, 2006) for (I); PLATON (Spek, 2009) and publCIF Westrip (2010) for (II).

\section{5-(2-Methylpropyl)-5-(prop-2-en-1-yl)-2-sulfanylidene-1,3-diazinane-4,6-dione (I)}

\section{Crystal data}

$\mathrm{C}_{11} \mathrm{H}_{16} \mathrm{~N}_{2} \mathrm{O}_{2} \mathrm{~S}$

$M_{r}=240.32$

Monoclinic, $P 2_{1} / n$

$a=8.7271(6) \AA$

$b=11.6521(4) \AA$

$c=12.5400(8) \AA$

$\beta=96.539(2)^{\circ}$

$V=1266.89(13) \AA^{3}$

$Z=4$

\section{Data collection}

Bruker-Nonius Roper CCD camera on $\kappa$ goniostat diffractometer

Radiation source: Bruker-Nonius FR591 rotating anode

Graphite monochromator

Detector resolution: 9.091 pixels $\mathrm{mm}^{-1}$

$\varphi \& \omega$ scans

Absorption correction: multi-scan

(SADABS; Sheldrick, 2007)

\section{Refinement}

Refinement on $F^{2}$

Least-squares matrix: full

$R\left[F^{2}>2 \sigma\left(F^{2}\right)\right]=0.045$

$w R\left(F^{2}\right)=0.108$

$S=1.04$
$F(000)=512$

$D_{\mathrm{x}}=1.260 \mathrm{Mg} \mathrm{m}^{-3}$

Mo $K \alpha$ radiation, $\lambda=0.71073 \AA$

Cell parameters from 10435 reflections

$\theta=2.9-27.5^{\circ}$

$\mu=0.24 \mathrm{~mm}^{-1}$

$T=120 \mathrm{~K}$

Prism, colourless

$0.40 \times 0.10 \times 0.05 \mathrm{~mm}$

$T_{\min }=0.924, T_{\max }=1.000$

9476 measured reflections

2519 independent reflections

1833 reflections with $I>2 \sigma(I)$

$R_{\text {int }}=0.067$

$\theta_{\text {max }}=26.4^{\circ}, \theta_{\min }=3.3^{\circ}$

$h=-9 \rightarrow 10$

$k=-12 \rightarrow 13$

$l=-15 \rightarrow 14$

2519 reflections

170 parameters

2 restraints

Primary atom site location: structure-invariant direct methods 
Secondary atom site location: difference Fourier map

Hydrogen site location: mixed

$\mathrm{H}$ atoms treated by a mixture of independent and constrained refinement

$w=1 /\left[\sigma^{2}\left(F_{\mathrm{o}}^{2}\right)+(0.0431 P)^{2}+0.281 P\right]$

where $P=\left(F_{\mathrm{o}}{ }^{2}+2 F_{\mathrm{c}}{ }^{2}\right) / 3$

\author{
$(\Delta / \sigma)_{\max }<0.001$ \\ $\Delta \rho_{\max }=0.31 \mathrm{e}^{-3}$ \\ $\Delta \rho_{\min }=-0.27$ e $\AA^{-3}$ \\ Extinction correction: SHELXL2014 \\ (Sheldrick, 2015b), \\ $\mathrm{Fc}^{*}=\mathrm{kFc}\left[1+0.001 \mathrm{xFc}^{2} \lambda^{3} / \sin (2 \theta)\right]^{-1 / 4}$ \\ Extinction coefficient: 0.011 (2)
}

Special details

Geometry. All esds (except the esd in the dihedral angle between two 1.s. planes) are estimated using the full covariance matrix. The cell esds are taken into account individually in the estimation of esds in distances, angles and torsion angles; correlations between esds in cell parameters are only used when they are defined by crystal symmetry. An approximate (isotropic) treatment of cell esds is used for estimating esds involving 1.s. planes.

Fractional atomic coordinates and isotropic or equivalent isotropic displacement parameters $\left(\AA^{2}\right)$

\begin{tabular}{|c|c|c|c|c|}
\hline & $x$ & $y$ & $z$ & $U_{\text {iso }} * / U_{\text {eq }}$ \\
\hline S2 & $0.13456(7)$ & $0.87633(4)$ & $0.94050(5)$ & 0.0259 (2) \\
\hline $\mathrm{O} 4$ & 0.42805 (18) & $0.66380(11)$ & $0.69496(13)$ & $0.0256(4)$ \\
\hline O6 & $0.26955(18)$ & $1.04475(11)$ & $0.60757(12)$ & $0.0261(4)$ \\
\hline N1 & $0.2119(2)$ & 0.95525 (14) & 0.75707 (14) & 0.0197 (4) \\
\hline H1 & $0.162(3)$ & $1.0169(17)$ & $0.771(2)$ & $0.036(7)^{*}$ \\
\hline N3 & $0.2876(2)$ & $0.76850(14)$ & $0.79944(14)$ & 0.0192 (4) \\
\hline H3 & $0.282(3)$ & $0.7101(16)$ & 0.8409 (17) & $0.030(7)^{*}$ \\
\hline $\mathrm{C} 2$ & 0.2138 (2) & 0.86637 (16) & $0.82860(17)$ & $0.0181(5)$ \\
\hline $\mathrm{C} 4$ & $0.3650(2)$ & $0.75482(16)$ & 0.71139 (17) & $0.0196(5)$ \\
\hline $\mathrm{C} 5$ & 0.3698 (2) & $0.85445(16)$ & 0.63413 (17) & 0.0187 (5) \\
\hline $\mathrm{C} 6$ & $0.2810(2)$ & 0.95949 (16) & $0.66426(17)$ & $0.0193(5)$ \\
\hline $\mathrm{C} 7$ & 0.2939 (3) & $0.81243(17)$ & $0.52224(17)$ & $0.0225(5)$ \\
\hline H7A & 0.3022 & 0.8740 & 0.4689 & $0.034(7)^{*}$ \\
\hline H7B & 0.3518 & 0.7451 & 0.5000 & $0.034(7)^{*}$ \\
\hline $\mathrm{C} 8$ & $0.1275(3)$ & 0.7801 (2) & $0.52146(18)$ & $0.0286(5)$ \\
\hline H8 & 0.0538 & 0.8398 & 0.5236 & $0.038(7)^{*}$ \\
\hline C9 & $0.0781(3)$ & $0.6733(2)$ & $0.5180(2)$ & $0.0397(7)$ \\
\hline H9A & 0.1494 & 0.6119 & 0.5157 & $0.054(9)^{*}$ \\
\hline H9B & -0.0287 & 0.6576 & 0.5176 & $0.051(8)^{*}$ \\
\hline $\mathrm{C} 10$ & 0.5409 (3) & $0.88428(17)$ & $0.62406(17)$ & $0.0221(5)$ \\
\hline $\mathrm{H} 10 \mathrm{~A}$ & 0.5867 & 0.8189 & 0.5884 & $0.029(6)^{*}$ \\
\hline H10B & 0.5423 & 0.9512 & 0.5756 & $0.025(6)^{*}$ \\
\hline C11 & $0.6456(3)$ & 0.9114 (2) & 0.7264 (2) & $0.0322(6)$ \\
\hline H11 & 0.6314 & 0.8501 & 0.7802 & $0.052(8)^{*}$ \\
\hline $\mathrm{C} 12$ & $0.6132(3)$ & $1.0262(3)$ & $0.7760(2)$ & $0.0494(8)$ \\
\hline $\mathrm{H} 12 \mathrm{~A}$ & 0.6195 & 1.0872 & 0.7228 & $0.074(11)^{*}$ \\
\hline H12B & 0.6895 & 1.0404 & 0.8382 & $0.076(10)^{*}$ \\
\hline $\mathrm{H} 12 \mathrm{C}$ & 0.5096 & 1.0254 & 0.7990 & $0.074(11)^{*}$ \\
\hline C13 & $0.8130(3)$ & 0.9075 (3) & 0.7015 (3) & $0.0484(8)$ \\
\hline $\mathrm{H} 13 \mathrm{~A}$ & 0.8366 & 0.8305 & 0.6764 & $0.068(10)^{*}$ \\
\hline H13B & 0.8819 & 0.9256 & 0.7666 & $0.069(10)^{*}$ \\
\hline $\mathrm{H} 13 \mathrm{C}$ & 0.8278 & 0.9639 & 0.6456 & $0.049(8)^{*}$ \\
\hline
\end{tabular}


Atomic displacement parameters $\left(\AA^{2}\right)$

\begin{tabular}{lllllll}
\hline & $U^{11}$ & $U^{22}$ & $U^{33}$ & $U^{12}$ & $U^{13}$ & $U^{23}$ \\
\hline S2 & $0.0311(4)$ & $0.0251(3)$ & $0.0237(3)$ & $0.0012(2)$ & $0.0123(3)$ & $0.0027(2)$ \\
O4 & $0.0280(9)$ & $0.0144(7)$ & $0.0365(10)$ & $0.0023(6)$ & $0.0129(7)$ & $0.0001(6)$ \\
O6 & $0.0392(10)$ & $0.0170(8)$ & $0.0238(9)$ & $0.0019(6)$ & $0.0113(7)$ & $0.0035(6)$ \\
N1 & $0.0239(11)$ & $0.0145(9)$ & $0.0222(10)$ & $0.0010(7)$ & $0.0086(8)$ & $0.0010(7)$ \\
N3 & $0.0234(10)$ & $0.0139(9)$ & $0.0212(10)$ & $0.0015(7)$ & $0.0063(8)$ & $0.0034(7)$ \\
C2 & $0.0164(11)$ & $0.0172(10)$ & $0.0203(11)$ & $-0.0026(8)$ & $0.0002(9)$ & $-0.0009(8)$ \\
C4 & $0.0164(11)$ & $0.0178(11)$ & $0.0248(12)$ & $-0.0042(8)$ & $0.0031(9)$ & $-0.0019(8)$ \\
C5 & $0.0214(12)$ & $0.0154(10)$ & $0.0205(11)$ & $-0.0015(8)$ & $0.0074(9)$ & $-0.0004(8)$ \\
C6 & $0.0222(12)$ & $0.0160(10)$ & $0.0200(12)$ & $-0.0020(8)$ & $0.0042(9)$ & $-0.0018(8)$ \\
C7 & $0.0262(13)$ & $0.0213(11)$ & $0.0207(12)$ & $-0.0024(9)$ & $0.0063(10)$ & $-0.0025(8)$ \\
C8 & $0.0259(13)$ & $0.0358(13)$ & $0.0240(13)$ & $-0.0013(10)$ & $0.0028(10)$ & $-0.0040(10)$ \\
C9 & $0.0355(16)$ & $0.0477(16)$ & $0.0343(15)$ & $-0.0172(13)$ & $-0.0032(12)$ & $0.0032(11)$ \\
C10 & $0.0238(12)$ & $0.0215(11)$ & $0.0225(12)$ & $-0.0024(8)$ & $0.0088(10)$ & $0.0005(8)$ \\
C11 & $0.0269(14)$ & $0.0405(14)$ & $0.0287(14)$ & $-0.0073(10)$ & $0.0016(11)$ & $0.0039(10)$ \\
C12 & $0.0388(18)$ & $0.068(2)$ & $0.0405(17)$ & $-0.0152(14)$ & $0.0031(14)$ & $-0.0245(15)$ \\
C13 & $0.0291(16)$ & $0.066(2)$ & $0.0496(19)$ & $-0.0035(13)$ & $0.0025(14)$ & $0.0078(15)$ \\
& & & & & & \\
\hline
\end{tabular}

Geometric parameters $\left(\AA,{ }^{\circ}\right)$

\begin{tabular}{|c|c|c|c|}
\hline $\mathrm{S} 2-\mathrm{C} 2$ & $1.638(2)$ & $\mathrm{C} 8-\mathrm{C} 9$ & $1.315(3)$ \\
\hline $\mathrm{O} 4-\mathrm{C} 4$ & $1.223(2)$ & $\mathrm{C} 8-\mathrm{H} 8$ & 0.9500 \\
\hline $\mathrm{O} 6-\mathrm{C} 6$ & $1.219(2)$ & C9-H9A & 0.9500 \\
\hline $\mathrm{N} 1-\mathrm{C} 2$ & $1.369(3)$ & C9-H9B & 0.9500 \\
\hline $\mathrm{N} 1-\mathrm{C} 6$ & $1.371(3)$ & $\mathrm{C} 10-\mathrm{C} 11$ & $1.522(3)$ \\
\hline $\mathrm{N} 1-\mathrm{H} 1$ & $0.867(16)$ & $\mathrm{C} 10-\mathrm{H} 10 \mathrm{~A}$ & 0.9900 \\
\hline $\mathrm{N} 3-\mathrm{C} 4$ & $1.367(3)$ & $\mathrm{C} 10-\mathrm{H} 10 \mathrm{~B}$ & 0.9900 \\
\hline $\mathrm{N} 3-\mathrm{C} 2$ & $1.379(3)$ & $\mathrm{C} 11-\mathrm{C} 12$ & $1.515(4)$ \\
\hline $\mathrm{N} 3-\mathrm{H} 3$ & $0.861(16)$ & $\mathrm{C} 11-\mathrm{C} 13$ & $1.529(4)$ \\
\hline $\mathrm{C} 4-\mathrm{C} 5$ & $1.516(3)$ & C11-H11 & 1.0000 \\
\hline $\mathrm{C} 5-\mathrm{C} 6$ & $1.519(3)$ & $\mathrm{C} 12-\mathrm{H} 12 \mathrm{~A}$ & 0.9800 \\
\hline $\mathrm{C} 5-\mathrm{C} 10$ & $1.552(3)$ & $\mathrm{C} 12-\mathrm{H} 12 \mathrm{~B}$ & 0.9800 \\
\hline $\mathrm{C} 5-\mathrm{C} 7$ & $1.561(3)$ & $\mathrm{C} 12-\mathrm{H} 12 \mathrm{C}$ & 0.9800 \\
\hline $\mathrm{C} 7-\mathrm{C} 8$ & $1.499(3)$ & C13-H13A & 0.9800 \\
\hline C7-H7A & 0.9900 & $\mathrm{C} 13-\mathrm{H} 13 \mathrm{~B}$ & 0.9800 \\
\hline C7-H7B & 0.9900 & $\mathrm{C} 13-\mathrm{H} 13 \mathrm{C}$ & 0.9800 \\
\hline $\mathrm{C} 2-\mathrm{N} 1-\mathrm{C} 6$ & 127.57 (17) & $\mathrm{C} 7-\mathrm{C} 8-\mathrm{H} 8$ & 118.3 \\
\hline $\mathrm{C} 2-\mathrm{N} 1-\mathrm{H} 1$ & $117.7(17)$ & $\mathrm{C} 8-\mathrm{C} 9-\mathrm{H} 9 \mathrm{~A}$ & 120.0 \\
\hline $\mathrm{C} 6-\mathrm{N} 1-\mathrm{H} 1$ & $114.8(17)$ & $\mathrm{C} 8-\mathrm{C} 9-\mathrm{H} 9 \mathrm{~B}$ & 120.0 \\
\hline $\mathrm{C} 4-\mathrm{N} 3-\mathrm{C} 2$ & $126.79(17)$ & $\mathrm{H} 9 \mathrm{~A}-\mathrm{C} 9-\mathrm{H} 9 \mathrm{~B}$ & 120.0 \\
\hline $\mathrm{C} 4-\mathrm{N} 3-\mathrm{H} 3$ & $117.7(16)$ & $\mathrm{C} 11-\mathrm{C} 10-\mathrm{C} 5$ & $117.95(18)$ \\
\hline $\mathrm{C} 2-\mathrm{N} 3-\mathrm{H} 3$ & $115.5(16)$ & $\mathrm{C} 11-\mathrm{C} 10-\mathrm{H} 10 \mathrm{~A}$ & 107.8 \\
\hline $\mathrm{N} 1-\mathrm{C} 2-\mathrm{N} 3$ & $115.02(18)$ & $\mathrm{C} 5-\mathrm{C} 10-\mathrm{H} 10 \mathrm{~A}$ & 107.8 \\
\hline $\mathrm{N} 1-\mathrm{C} 2-\mathrm{S} 2$ & $122.21(15)$ & $\mathrm{C} 11-\mathrm{C} 10-\mathrm{H} 10 \mathrm{~B}$ & 107.8 \\
\hline $\mathrm{N} 3-\mathrm{C} 2-\mathrm{S} 2$ & $122.77(15)$ & $\mathrm{C} 5-\mathrm{C} 10-\mathrm{H} 10 \mathrm{~B}$ & 107.8 \\
\hline
\end{tabular}




\begin{tabular}{|c|c|c|c|}
\hline $\mathrm{O} 4-\mathrm{C} 4-\mathrm{N} 3$ & $120.68(18)$ & $\mathrm{H} 10 \mathrm{~A}-\mathrm{C} 10-\mathrm{H} 10 \mathrm{~B}$ & 107.2 \\
\hline $\mathrm{O} 4-\mathrm{C} 4-\mathrm{C} 5$ & $120.70(19)$ & $\mathrm{C} 12-\mathrm{C} 11-\mathrm{C} 10$ & $114.0(2)$ \\
\hline $\mathrm{N} 3-\mathrm{C} 4-\mathrm{C} 5$ & $118.62(17)$ & $\mathrm{C} 12-\mathrm{C} 11-\mathrm{C} 13$ & $109.7(2)$ \\
\hline $\mathrm{C} 4-\mathrm{C} 5-\mathrm{C} 6$ & $113.95(17)$ & $\mathrm{C} 10-\mathrm{C} 11-\mathrm{C} 13$ & $108.5(2)$ \\
\hline $\mathrm{C} 4-\mathrm{C} 5-\mathrm{C} 10$ & $108.69(17)$ & $\mathrm{C} 12-\mathrm{C} 11-\mathrm{H} 11$ & 108.1 \\
\hline $\mathrm{C} 6-\mathrm{C} 5-\mathrm{C} 10$ & $111.26(16)$ & $\mathrm{C} 10-\mathrm{C} 11-\mathrm{H} 11$ & 108.1 \\
\hline $\mathrm{C} 4-\mathrm{C} 5-\mathrm{C} 7$ & $107.13(16)$ & $\mathrm{C} 13-\mathrm{C} 11-\mathrm{H} 11$ & 108.1 \\
\hline $\mathrm{C} 6-\mathrm{C} 5-\mathrm{C} 7$ & $107.46(17)$ & $\mathrm{C} 11-\mathrm{C} 12-\mathrm{H} 12 \mathrm{~A}$ & 109.5 \\
\hline $\mathrm{C} 10-\mathrm{C} 5-\mathrm{C} 7$ & $108.11(16)$ & $\mathrm{C} 11-\mathrm{C} 12-\mathrm{H} 12 \mathrm{~B}$ & 109.5 \\
\hline $\mathrm{O} 6-\mathrm{C} 6-\mathrm{N} 1$ & $120.64(18)$ & $\mathrm{H} 12 \mathrm{~A}-\mathrm{C} 12-\mathrm{H} 12 \mathrm{~B}$ & 109.5 \\
\hline $\mathrm{O} 6-\mathrm{C} 6-\mathrm{C} 5$ & $121.44(18)$ & $\mathrm{C} 11-\mathrm{C} 12-\mathrm{H} 12 \mathrm{C}$ & 109.5 \\
\hline $\mathrm{N} 1-\mathrm{C} 6-\mathrm{C} 5$ & $117.91(17)$ & $\mathrm{H} 12 \mathrm{~A}-\mathrm{C} 12-\mathrm{H} 12 \mathrm{C}$ & 109.5 \\
\hline $\mathrm{C} 8-\mathrm{C} 7-\mathrm{C} 5$ & $113.35(17)$ & $\mathrm{H} 12 \mathrm{~B}-\mathrm{C} 12-\mathrm{H} 12 \mathrm{C}$ & 109.5 \\
\hline $\mathrm{C} 8-\mathrm{C} 7-\mathrm{H} 7 \mathrm{~A}$ & 108.9 & $\mathrm{C} 11-\mathrm{C} 13-\mathrm{H} 13 \mathrm{~A}$ & 109.5 \\
\hline $\mathrm{C} 5-\mathrm{C} 7-\mathrm{H} 7 \mathrm{~A}$ & 108.9 & $\mathrm{C} 11-\mathrm{C} 13-\mathrm{H} 13 \mathrm{~B}$ & 109.5 \\
\hline $\mathrm{C} 8-\mathrm{C} 7-\mathrm{H} 7 \mathrm{~B}$ & 108.9 & $\mathrm{H} 13 \mathrm{~A}-\mathrm{C} 13-\mathrm{H} 13 \mathrm{~B}$ & 109.5 \\
\hline $\mathrm{C} 5-\mathrm{C} 7-\mathrm{H} 7 \mathrm{~B}$ & 108.9 & $\mathrm{C} 11-\mathrm{C} 13-\mathrm{H} 13 \mathrm{C}$ & 109.5 \\
\hline $\mathrm{H} 7 \mathrm{~A}-\mathrm{C} 7-\mathrm{H} 7 \mathrm{~B}$ & 107.7 & $\mathrm{H} 13 \mathrm{~A}-\mathrm{C} 13-\mathrm{H} 13 \mathrm{C}$ & 109.5 \\
\hline $\mathrm{C} 9-\mathrm{C} 8-\mathrm{C} 7$ & $123.4(2)$ & $\mathrm{H} 13 \mathrm{~B}-\mathrm{C} 13-\mathrm{H} 13 \mathrm{C}$ & 109.5 \\
\hline $\mathrm{C} 9-\mathrm{C} 8-\mathrm{H} 8$ & 118.3 & & \\
\hline $\mathrm{C} 6-\mathrm{N} 1-\mathrm{C} 2-\mathrm{N} 3$ & $-3.7(3)$ & $\mathrm{C} 10-\mathrm{C} 5-\mathrm{C} 6-\mathrm{O} 6$ & $59.5(3)$ \\
\hline $\mathrm{C} 6-\mathrm{N} 1-\mathrm{C} 2-\mathrm{S} 2$ & $176.16(17)$ & $\mathrm{C} 7-\mathrm{C} 5-\mathrm{C} 6-\mathrm{O} 6$ & $-58.7(2)$ \\
\hline $\mathrm{C} 4-\mathrm{N} 3-\mathrm{C} 2-\mathrm{N} 1$ & $4.2(3)$ & $\mathrm{C} 4-\mathrm{C} 5-\mathrm{C} 6-\mathrm{N} 1$ & $2.0(3)$ \\
\hline $\mathrm{C} 4-\mathrm{N} 3-\mathrm{C} 2-\mathrm{S} 2$ & $-175.64(17)$ & $\mathrm{C} 10-\mathrm{C} 5-\mathrm{C} 6-\mathrm{N} 1$ & $-121.3(2)$ \\
\hline $\mathrm{C} 2-\mathrm{N} 3-\mathrm{C} 4-\mathrm{O} 4$ & $178.9(2)$ & $\mathrm{C} 7-\mathrm{C} 5-\mathrm{C} 6-\mathrm{N} 1$ & $120.6(2)$ \\
\hline $\mathrm{C} 2-\mathrm{N} 3-\mathrm{C} 4-\mathrm{C} 5$ & $-1.6(3)$ & $\mathrm{C} 4-\mathrm{C} 5-\mathrm{C} 7-\mathrm{C} 8$ & $62.7(2)$ \\
\hline $\mathrm{O} 4-\mathrm{C} 4-\mathrm{C} 5-\mathrm{C} 6$ & $177.91(19)$ & $\mathrm{C} 6-\mathrm{C} 5-\mathrm{C} 7-\mathrm{C} 8$ & $-60.1(2)$ \\
\hline $\mathrm{N} 3-\mathrm{C} 4-\mathrm{C} 5-\mathrm{C} 6$ & $-1.6(3)$ & $\mathrm{C} 10-\mathrm{C} 5-\mathrm{C} 7-\mathrm{C} 8$ & $179.67(17)$ \\
\hline $\mathrm{O} 4-\mathrm{C} 4-\mathrm{C} 5-\mathrm{C} 10$ & $-57.4(2)$ & $\mathrm{C} 5-\mathrm{C} 7-\mathrm{C} 8-\mathrm{C} 9$ & $-106.1(3)$ \\
\hline $\mathrm{N} 3-\mathrm{C} 4-\mathrm{C} 5-\mathrm{C} 10$ & $123.1(2)$ & $\mathrm{C} 4-\mathrm{C} 5-\mathrm{C} 10-\mathrm{C} 11$ & $-55.6(2)$ \\
\hline $\mathrm{O} 4-\mathrm{C} 4-\mathrm{C} 5-\mathrm{C} 7$ & $59.2(3)$ & $\mathrm{C} 6-\mathrm{C} 5-\mathrm{C} 10-\mathrm{C} 11$ & $70.6(2)$ \\
\hline $\mathrm{N} 3-\mathrm{C} 4-\mathrm{C} 5-\mathrm{C} 7$ & $-120.3(2)$ & $\mathrm{C} 7-\mathrm{C} 5-\mathrm{C} 10-\mathrm{C} 11$ & $-171.58(18)$ \\
\hline $\mathrm{C} 2-\mathrm{N} 1-\mathrm{C} 6-\mathrm{O} 6$ & $179.9(2)$ & $\mathrm{C} 5-\mathrm{C} 10-\mathrm{C} 11-\mathrm{C} 12$ & $-71.7(3)$ \\
\hline $\mathrm{C} 2-\mathrm{N} 1-\mathrm{C} 6-\mathrm{C} 5$ & $0.6(3)$ & $\mathrm{C} 5-\mathrm{C} 10-\mathrm{C} 11-\mathrm{C} 13$ & $165.64(19)$ \\
\hline $\mathrm{C} 4-\mathrm{C} 5-\mathrm{C} 6-\mathrm{O} 6$ & $-177.20(19)$ & & \\
\hline
\end{tabular}

Hydrogen-bond geometry $\left(\AA,{ }^{\circ}\right)$

\begin{tabular}{lllll}
\hline$D-\mathrm{H} \cdots A$ & $D-\mathrm{H}$ & $\mathrm{H} \cdots A$ & $D \cdots A$ & $D-\mathrm{H} \cdots A$ \\
\hline $\mathrm{N} 1-\mathrm{H} 1 \cdots \mathrm{O} 4^{\mathrm{i}}$ & $0.87(2)$ & $1.95(2)$ & $2.815(2)$ & $174(2)$ \\
$\mathrm{N} 3-\mathrm{H} 3 \cdots 6^{\mathrm{ii}}$ & $0.86(2)$ & $2.10(2)$ & $2.922(2)$ & $160(2)$ \\
\hline
\end{tabular}

Symmetry codes: (i) $-x+1 / 2, y+1 / 2,-z+3 / 2$; (ii) $-x+1 / 2, y-1 / 2,-z+3 / 2$. 
5-(1-Methylbutyl)-5-[2-(methylsulfanyl)ethyl]-2-sulfanylidene-1,3-diazinane-4,6-dione (II)

Crystal data

$\mathrm{C}_{12} \mathrm{H}_{20} \mathrm{~N}_{2} \mathrm{O}_{2} \mathrm{~S}_{2}$

$M_{r}=288.42$

Monoclinic, $C 2 / c$

$a=15.1873(2) \AA$

$b=9.0920(1) \AA$

$c=20.8684(3) \AA$

$\beta=96.083(1)^{\circ}$

$V=2865.34(6) \AA^{3}$

$Z=8$

Data collection

Bruker-Nonius APEXII CCD camera on $\kappa$ goniostat diffractometer

Radiation source: Bruker-Nonius FR591 rotating anode

$10 \mathrm{~cm}$ confocal mirrors monochromator

Detector resolution: 9.091 pixels $\mathrm{mm}^{-1}$

$\varphi \& \omega$ scans

Absorption correction: multi-scan

(SADABS; Sheldrick, 2007)

Refinement

Refinement on $F^{2}$

Least-squares matrix: full

$R\left[F^{2}>2 \sigma\left(F^{2}\right)\right]=0.038$

$w R\left(F^{2}\right)=0.084$

$S=1.14$

2813 reflections

192 parameters

2 restraints
$F(000)=1232$

$D_{\mathrm{x}}=1.337 \mathrm{Mg} \mathrm{m}^{-3}$

Mo $K \alpha$ radiation, $\lambda=0.71073 \AA$

Cell parameters from 29667 reflections

$\theta=2.9-27.5^{\circ}$

$\mu=0.37 \mathrm{~mm}^{-1}$

$T=120 \mathrm{~K}$

Block, colourless

$0.15 \times 0.15 \times 0.10 \mathrm{~mm}$

$T_{\min }=0.974, T_{\max }=1.000$

24772 measured reflections

2813 independent reflections

2630 reflections with $I>2 \sigma(I)$

$R_{\text {int }}=0.034$

$\theta_{\max }=26.0^{\circ}, \theta_{\min }=3.2^{\circ}$

$h=-18 \rightarrow 18$

$k=-11 \rightarrow 11$

$l=-25 \rightarrow 25$

Hydrogen site location: mixed

$\mathrm{H}$ atoms treated by a mixture of independent and constrained refinement

$w=1 /\left[\sigma^{2}\left(F_{\mathrm{o}}^{2}\right)+(0.0192 P)^{2}+6.3729 P\right]$

where $P=\left(F_{\mathrm{o}}^{2}+2 F_{\mathrm{c}}^{2}\right) / 3$

$(\Delta / \sigma)_{\max }=0.001$

$\Delta \rho_{\max }=0.53 \mathrm{e} \AA^{-3}$

$\Delta \rho_{\min }=-0.29 \mathrm{e}^{-3}$

Special details

Geometry. All esds (except the esd in the dihedral angle between two 1.s. planes) are estimated using the full covariance matrix. The cell esds are taken into account individually in the estimation of esds in distances, angles and torsion angles; correlations between esds in cell parameters are only used when they are defined by crystal symmetry. An approximate (isotropic) treatment of cell esds is used for estimating esds involving l.s. planes.

Fractional atomic coordinates and isotropic or equivalent isotropic displacement parameters $\left(\AA^{2}\right)$

\begin{tabular}{lllll}
\hline & $x$ & $y$ & $z$ & $U_{\text {iso }} * / U_{\text {eq }}$ \\
\hline S2 & $-0.17769(3)$ & $0.62565(6)$ & $0.34779(2)$ & $0.02411(13)$ \\
S9 & $0.14236(3)$ & $1.10685(5)$ & $0.51192(2)$ & $0.02392(13)$ \\
O4 & $0.09157(9)$ & $0.87624(15)$ & $0.30012(6)$ & $0.0218(3)$ \\
O6 & $0.09747(9)$ & $0.59147(14)$ & $0.49086(6)$ & $0.0220(3)$ \\
N1 & $-0.02312(10)$ & $0.60656(17)$ & $0.41868(7)$ & $0.0177(3)$ \\
H1 & $-0.0510(14)$ & $0.551(2)$ & $0.4430(10)$ & $0.031(6)^{*}$ \\
N3 & $-0.02774(10)$ & $0.75738(17)$ & $0.32870(7)$ & $0.0181(3)$ \\
H3 & $-0.0574(14)$ & $0.798(2)$ & $0.2962(9)$ & $0.029(6)^{*}$ \\
C2 & $-0.07264(12)$ & $0.6645(2)$ & $0.36566(8)$ & $0.0173(4)$
\end{tabular}




$\begin{array}{lllll}\text { C4 } & 0.06055(12) & 0.79499(19) & 0.33818(8) & 0.0167(4) \\ \text { C6 } & 0.06356(12) & 0.63907(19) & 0.43924(8) & 0.0171(4) \\ \text { C7 } & 0.16068(12) & 0.85980(19) & 0.43567(8) & 0.0161(4) \\ \text { H7A } & 0.2088 & 0.8208 & 0.4667 & 0.020(5)^{*} \\ \text { H7B } & 0.1875 & 0.9281 & 0.4063 & 0.021(5)^{*} \\ \text { C5 } & 0.11666(11) & 0.73082(19) & 0.39593(8) & 0.0158(4) \\ \text { C8 } & 0.09525(13) & 0.9443(2) & 0.47235(9) & 0.0218(4) \\ \text { H8A } & 0.0735 & 0.8786 & 0.5051 & 0.029(6)^{*} \\ \text { H8B } & 0.0437 & 0.9733 & 0.4419 & 0.031(6)^{*} \\ \text { C10 } & 0.13117(16) & 1.2337(2) & 0.44571(12) & 0.0345(5) \\ \text { H10A } & 0.1605 & 1.1935 & 0.4099 & 0.071(10)^{*} \\ \text { H10B } & 0.1586 & 1.3277 & 0.4594 & 0.053(8)^{*} \\ \text { H10C } & 0.0682 & 1.2492 & 0.4316 & 0.047(8)^{*} \\ \text { C12 } & 0.18817(13) & 0.6231(2) & 0.37204(9) & 0.0217(4) \\ \text { H12 } & 0.2155 & 0.5694 & 0.4110 & 0.033(6)^{*} \\ \text { C13 } & 0.26315(13) & 0.7017(2) & 0.34349(10) & 0.0258(4) \\ \text { H13A } & 0.2880 & 0.7777 & 0.3742 & 0.040(7)^{*} \\ \text { H13B } & 0.2390 & 0.7523 & 0.3034 & 0.043(7)^{*} \\ \text { C14 } & 0.33787(14) & 0.5984(2) & 0.32810(11) & 0.0308(5) \\ \text { H14A } & 0.3564 & 0.5368 & 0.3662 & 0.029(6)^{*} \\ \text { H14B } & 0.3159 & 0.5324 & 0.2922 & 0.054(8)^{*} \\ \text { C15 } & 0.41659(17) & 0.6846(3) & 0.30960(13) & 0.0438(6) \\ \text { H15A } & 0.3988 & 0.7425 & 0.2708 & 0.072(10)^{*} \\ \text { H15B } & 0.4640 & 0.6164 & 0.3011 & 0.053(8)^{*} \\ \text { H15C } & 0.4380 & 0.7508 & 0.3449 & 0.073(10)^{*} \\ \text { C16 } & 0.14371(14) & 0.5061(2) & 0.32611(9) & 0.0250(4) \\ \text { H16A } & 0.1226 & 0.5521 & 0.2849 & 0.036(7)^{*} \\ \text { H16B } & 0.0935 & 0.4628 & 0.3452 & 0.038(7)^{*} \\ \text { H16C } & 0.1867 & 0.4290 & 0.3190 & 0.040(7)^{*}\end{array}$

Atomic displacement parameters $\left(\AA^{2}\right)$

\begin{tabular}{lllllll}
\hline & $U^{11}$ & $U^{22}$ & $U^{33}$ & $U^{12}$ & $U^{13}$ & $U^{23}$ \\
\hline S2 & $0.0188(2)$ & $0.0307(3)$ & $0.0228(2)$ & $-0.0057(2)$ & $0.00256(18)$ & $0.0002(2)$ \\
S9 & $0.0251(3)$ & $0.0196(2)$ & $0.0270(3)$ & $-0.00207(19)$ & $0.00215(19)$ & $-0.00781(19)$ \\
O4 & $0.0228(7)$ & $0.0243(7)$ & $0.0184(6)$ & $-0.0061(6)$ & $0.0029(5)$ & $0.0052(5)$ \\
O6 & $0.0248(7)$ & $0.0210(7)$ & $0.0195(7)$ & $-0.0020(6)$ & $-0.0006(5)$ & $0.0056(5)$ \\
N1 & $0.0194(8)$ & $0.0159(7)$ & $0.0183(8)$ & $-0.0032(6)$ & $0.0045(6)$ & $0.0025(6)$ \\
N3 & $0.0177(8)$ & $0.0212(8)$ & $0.0152(7)$ & $-0.0008(6)$ & $0.0009(6)$ & $0.0030(6)$ \\
C2 & $0.0211(9)$ & $0.0159(9)$ & $0.0152(8)$ & $-0.0005(7)$ & $0.0038(7)$ & $-0.0027(7)$ \\
C4 & $0.0200(9)$ & $0.0157(9)$ & $0.0145(8)$ & $0.0003(7)$ & $0.0028(7)$ & $-0.0030(7)$ \\
C6 & $0.0209(9)$ & $0.0119(8)$ & $0.0186(9)$ & $-0.0001(7)$ & $0.0029(7)$ & $-0.0018(7)$ \\
C7 & $0.0164(9)$ & $0.0147(8)$ & $0.0172(8)$ & $-0.0012(7)$ & $0.0023(7)$ & $-0.0011(7)$ \\
C5 & $0.0153(8)$ & $0.0155(8)$ & $0.0169(8)$ & $0.0004(7)$ & $0.0026(7)$ & $0.0000(7)$ \\
C8 & $0.0230(10)$ & $0.0169(9)$ & $0.0266(10)$ & $-0.0043(8)$ & $0.0081(8)$ & $-0.0067(8)$ \\
C10 & $0.0388(13)$ & $0.0191(10)$ & $0.0469(13)$ & $0.0002(9)$ & $0.0106(11)$ & $0.0060(10)$ \\
C12 & $0.0222(10)$ & $0.0183(9)$ & $0.0250(10)$ & $0.0029(8)$ & $0.0047(8)$ & $-0.0032(8)$ \\
C13 & $0.0249(10)$ & $0.0237(10)$ & $0.0299(10)$ & $0.0025(8)$ & $0.0081(8)$ & $0.0006(8)$
\end{tabular}




\begin{tabular}{lllllll}
$\mathrm{C} 14$ & $0.0283(11)$ & $0.0317(12)$ & $0.0345(12)$ & $0.0080(9)$ & $0.0122(9)$ & $-0.0006(9)$ \\
$\mathrm{C} 15$ & $0.0387(14)$ & $0.0430(14)$ & $0.0528(15)$ & $0.0091(12)$ & $0.0189(12)$ & $0.0070(13)$ \\
$\mathrm{C} 16$ & $0.0312(11)$ & $0.0189(9)$ & $0.0258(10)$ & $0.0007(8)$ & $0.0078(8)$ & $-0.0048(8)$ \\
\hline
\end{tabular}

Geometric parameters $\left(\stackrel{A}{\circ}{ }^{\circ}\right)$

\begin{tabular}{|c|c|c|c|}
\hline $\mathrm{S} 2-\mathrm{C} 2$ & $1.6381(19)$ & $\mathrm{C} 8-\mathrm{H} 8 \mathrm{~B}$ & 0.9900 \\
\hline $\mathrm{S} 9-\mathrm{C} 10$ & $1.794(2)$ & $\mathrm{C} 10-\mathrm{H} 10 \mathrm{~A}$ & 0.9800 \\
\hline $\mathrm{S} 9-\mathrm{C} 8$ & $1.8033(19)$ & $\mathrm{C} 10-\mathrm{H} 10 \mathrm{~B}$ & 0.9800 \\
\hline $\mathrm{O} 4-\mathrm{C} 4$ & $1.216(2)$ & $\mathrm{C} 10-\mathrm{H} 10 \mathrm{C}$ & 0.9800 \\
\hline $\mathrm{O} 6-\mathrm{C} 6$ & $1.223(2)$ & $\mathrm{C} 12-\mathrm{C} 13$ & $1.519(3)$ \\
\hline $\mathrm{N} 1-\mathrm{C} 6$ & $1.373(2)$ & $\mathrm{C} 12-\mathrm{C} 16$ & $1.539(3)$ \\
\hline $\mathrm{N} 1-\mathrm{C} 2$ & $1.375(2)$ & $\mathrm{C} 12-\mathrm{H} 12$ & 1.0000 \\
\hline $\mathrm{N} 1-\mathrm{H} 1$ & $0.858(16)$ & $\mathrm{C} 13-\mathrm{C} 14$ & $1.533(3)$ \\
\hline $\mathrm{N} 3-\mathrm{C} 2$ & $1.373(2)$ & $\mathrm{C} 13-\mathrm{H} 13 \mathrm{~A}$ & 0.9900 \\
\hline $\mathrm{N} 3-\mathrm{C} 4$ & $1.378(2)$ & $\mathrm{C} 13-\mathrm{H} 13 \mathrm{~B}$ & 0.9900 \\
\hline $\mathrm{N} 3-\mathrm{H} 3$ & $0.856(16)$ & $\mathrm{C} 14-\mathrm{C} 15$ & $1.513(3)$ \\
\hline $\mathrm{C} 4-\mathrm{C} 5$ & $1.517(2)$ & $\mathrm{C} 14-\mathrm{H} 14 \mathrm{~A}$ & 0.9900 \\
\hline $\mathrm{C} 6-\mathrm{C} 5$ & $1.522(2)$ & $\mathrm{C} 14-\mathrm{H} 14 \mathrm{~B}$ & 0.9900 \\
\hline $\mathrm{C} 7-\mathrm{C} 8$ & $1.525(2)$ & C15-H15A & 0.9800 \\
\hline $\mathrm{C} 7-\mathrm{C} 5$ & $1.547(2)$ & C15-H15B & 0.9800 \\
\hline C7-H7A & 0.9900 & $\mathrm{C} 15-\mathrm{H} 15 \mathrm{C}$ & 0.9800 \\
\hline C7-H7B & 0.9900 & $\mathrm{C} 16-\mathrm{H} 16 \mathrm{~A}$ & 0.9800 \\
\hline $\mathrm{C} 5-\mathrm{C} 12$ & $1.582(2)$ & C16-H16B & 0.9800 \\
\hline $\mathrm{C} 8-\mathrm{H} 8 \mathrm{~A}$ & 0.9900 & $\mathrm{C} 16-\mathrm{H} 16 \mathrm{C}$ & 0.9800 \\
\hline $\mathrm{C} 10-\mathrm{S} 9-\mathrm{C} 8$ & $99.99(10)$ & $\mathrm{S} 9-\mathrm{C} 10-\mathrm{H} 10 \mathrm{~B}$ & 109.5 \\
\hline $\mathrm{C} 6-\mathrm{N} 1-\mathrm{C} 2$ & $126.40(15)$ & $\mathrm{H} 10 \mathrm{~A}-\mathrm{C} 10-\mathrm{H} 10 \mathrm{~B}$ & 109.5 \\
\hline $\mathrm{C} 6-\mathrm{N} 1-\mathrm{H} 1$ & $117.3(16)$ & $\mathrm{S} 9-\mathrm{C} 10-\mathrm{H} 10 \mathrm{C}$ & 109.5 \\
\hline $\mathrm{C} 2-\mathrm{N} 1-\mathrm{H} 1$ & $116.1(16)$ & $\mathrm{H} 10 \mathrm{~A}-\mathrm{C} 10-\mathrm{H} 10 \mathrm{C}$ & 109.5 \\
\hline $\mathrm{C} 2-\mathrm{N} 3-\mathrm{C} 4$ & $127.29(16)$ & $\mathrm{H} 10 \mathrm{~B}-\mathrm{C} 10-\mathrm{H} 10 \mathrm{C}$ & 109.5 \\
\hline $\mathrm{C} 2-\mathrm{N} 3-\mathrm{H} 3$ & $117.3(16)$ & $\mathrm{C} 13-\mathrm{C} 12-\mathrm{C} 16$ & $112.25(16)$ \\
\hline $\mathrm{C} 4-\mathrm{N} 3-\mathrm{H} 3$ & $115.4(16)$ & $\mathrm{C} 13-\mathrm{C} 12-\mathrm{C} 5$ & $113.62(15)$ \\
\hline $\mathrm{N} 3-\mathrm{C} 2-\mathrm{N} 1$ & $115.21(16)$ & $\mathrm{C} 16-\mathrm{C} 12-\mathrm{C} 5$ & $110.71(15)$ \\
\hline $\mathrm{N} 3-\mathrm{C} 2-\mathrm{S} 2$ & $122.31(14)$ & $\mathrm{C} 13-\mathrm{C} 12-\mathrm{H} 12$ & 106.6 \\
\hline $\mathrm{N} 1-\mathrm{C} 2-\mathrm{S} 2$ & $122.47(14)$ & $\mathrm{C} 16-\mathrm{C} 12-\mathrm{H} 12$ & 106.6 \\
\hline $\mathrm{O} 4-\mathrm{C} 4-\mathrm{N} 3$ & $119.72(16)$ & $\mathrm{C} 5-\mathrm{C} 12-\mathrm{H} 12$ & 106.6 \\
\hline $\mathrm{O} 4-\mathrm{C} 4-\mathrm{C} 5$ & $121.92(16)$ & $\mathrm{C} 12-\mathrm{C} 13-\mathrm{C} 14$ & $113.35(17)$ \\
\hline $\mathrm{N} 3-\mathrm{C} 4-\mathrm{C} 5$ & $118.36(15)$ & $\mathrm{C} 12-\mathrm{C} 13-\mathrm{H} 13 \mathrm{~A}$ & 108.9 \\
\hline $\mathrm{O} 6-\mathrm{C} 6-\mathrm{N} 1$ & $119.97(16)$ & $\mathrm{C} 14-\mathrm{C} 13-\mathrm{H} 13 \mathrm{~A}$ & 108.9 \\
\hline $\mathrm{O} 6-\mathrm{C} 6-\mathrm{C} 5$ & $121.12(16)$ & $\mathrm{C} 12-\mathrm{C} 13-\mathrm{H} 13 \mathrm{~B}$ & 108.9 \\
\hline $\mathrm{N} 1-\mathrm{C} 6-\mathrm{C} 5$ & $118.89(15)$ & $\mathrm{C} 14-\mathrm{C} 13-\mathrm{H} 13 \mathrm{~B}$ & 108.9 \\
\hline $\mathrm{C} 8-\mathrm{C} 7-\mathrm{C} 5$ & $112.56(14)$ & $\mathrm{H} 13 \mathrm{~A}-\mathrm{C} 13-\mathrm{H} 13 \mathrm{~B}$ & 107.7 \\
\hline $\mathrm{C} 8-\mathrm{C} 7-\mathrm{H} 7 \mathrm{~A}$ & 109.1 & $\mathrm{C} 15-\mathrm{C} 14-\mathrm{C} 13$ & $111.01(19)$ \\
\hline $\mathrm{C} 5-\mathrm{C} 7-\mathrm{H} 7 \mathrm{~A}$ & 109.1 & $\mathrm{C} 15-\mathrm{C} 14-\mathrm{H} 14 \mathrm{~A}$ & 109.4 \\
\hline $\mathrm{C} 8-\mathrm{C} 7-\mathrm{H} 7 \mathrm{~B}$ & 109.1 & $\mathrm{C} 13-\mathrm{C} 14-\mathrm{H} 14 \mathrm{~A}$ & 109.4 \\
\hline $\mathrm{C} 5-\mathrm{C} 7-\mathrm{H} 7 \mathrm{~B}$ & 109.1 & $\mathrm{C} 15-\mathrm{C} 14-\mathrm{H} 14 \mathrm{~B}$ & 109.4 \\
\hline $\mathrm{H} 7 \mathrm{~A}-\mathrm{C} 7-\mathrm{H} 7 \mathrm{~B}$ & 107.8 & $\mathrm{C} 13-\mathrm{C} 14-\mathrm{H} 14 \mathrm{~B}$ & 109.4 \\
\hline
\end{tabular}




$\mathrm{C} 4-\mathrm{C} 5-\mathrm{C} 6$
$\mathrm{C} 4-\mathrm{C} 5-\mathrm{C} 7$
$\mathrm{C} 6-\mathrm{C} 5-\mathrm{C} 7$
$\mathrm{C} 4-\mathrm{C} 5-\mathrm{C} 12$
$\mathrm{C} 6-\mathrm{C} 5-\mathrm{C} 12$
$\mathrm{C} 7-\mathrm{C} 5-\mathrm{C} 12$
$\mathrm{C} 7-\mathrm{C} 8-\mathrm{S} 9$
$\mathrm{C} 7-\mathrm{C} 8-\mathrm{H} 8 \mathrm{~A}$
$\mathrm{~S} 9-\mathrm{C} 8-\mathrm{H} 8 \mathrm{~A}$
$\mathrm{C} 7-\mathrm{C} 8-\mathrm{H} 8 \mathrm{~B}$
$\mathrm{~S} 9-\mathrm{C} 8-\mathrm{H} 8 \mathrm{~B}$
$\mathrm{H} 8 \mathrm{~A}-\mathrm{C} 8-\mathrm{H} 8 \mathrm{~B}$
$\mathrm{~S} 9-\mathrm{C} 10-\mathrm{H} 10 \mathrm{~A}$

$\mathrm{C} 4-\mathrm{N} 3-\mathrm{C} 2-\mathrm{N} 1$
$\mathrm{C} 4-\mathrm{N} 3-\mathrm{C} 2-\mathrm{S} 2$
$\mathrm{C} 6-\mathrm{N} 1-\mathrm{C} 2-\mathrm{N} 3$
$\mathrm{C} 6-\mathrm{N} 1-\mathrm{C} 2-\mathrm{S} 2$
$\mathrm{C} 2-\mathrm{N} 3-\mathrm{C} 4-\mathrm{O} 4$
$\mathrm{C} 2-\mathrm{N} 3-\mathrm{C} 4-\mathrm{C} 5$
$\mathrm{C} 2-\mathrm{N} 1-\mathrm{C} 6-\mathrm{O} 6$
$\mathrm{C} 2-\mathrm{N} 1-\mathrm{C} 6-\mathrm{C} 5$
$\mathrm{O} 4-\mathrm{C} 4-\mathrm{C} 5-\mathrm{C} 6$
$\mathrm{~N} 3-\mathrm{C} 4-\mathrm{C} 5-\mathrm{C} 6$
$\mathrm{O} 4-\mathrm{C} 4-\mathrm{C} 5-\mathrm{C} 7$
$\mathrm{~N} 3-\mathrm{C} 4-\mathrm{C} 5-\mathrm{C} 7$
$\mathrm{O} 4-\mathrm{C} 4-\mathrm{C} 5-\mathrm{C} 12$
$\mathrm{~N} 3-\mathrm{C} 4-\mathrm{C} 5-\mathrm{C} 12$
$\mathrm{O} 6-\mathrm{C} 6-\mathrm{C} 5-\mathrm{C} 4$
$\mathrm{~N} 1-\mathrm{C} 6-\mathrm{C} 5-\mathrm{C} 4$
$\mathrm{O} 6-\mathrm{C} 6-\mathrm{C} 5-\mathrm{C} 7$

$113.21(15)$

$107.99(14)$

$108.86(14)$

$109.53(14)$

$105.81(14)$

$111.49(14)$

$113.25(13)$

108.9

108.9

108.9

108.9

107.7

109.5

$1.7(3)$

$-178.33(14)$

$3.8(3)$

$-176.19(14)$

$178.09(17)$

$-1.0(3)$

$172.51(17)$

$-9.3(3)$

$176.79(16)$

$-4.2(2)$

$56.2(2)$

$-124.77(16)$

$-65.4(2)$

$113.64(17)$

$-173.00(16)$

$8.8(2)$

$-52.9(2)$

$$
\begin{aligned}
& \mathrm{H} 14 \mathrm{~A}-\mathrm{C} 14-\mathrm{H} 14 \mathrm{~B} \\
& \mathrm{C} 14-\mathrm{C} 15-\mathrm{H} 15 \mathrm{~A} \\
& \mathrm{C} 14-\mathrm{C} 15-\mathrm{H} 15 \mathrm{~B} \\
& \mathrm{H} 15 \mathrm{~A}-\mathrm{C} 15-\mathrm{H} 15 \mathrm{~B} \\
& \mathrm{C} 14-\mathrm{C} 15-\mathrm{H} 15 \mathrm{C} \\
& \mathrm{H} 15 \mathrm{~A}-\mathrm{C} 15-\mathrm{H} 15 \mathrm{C} \\
& \mathrm{H} 15 \mathrm{~B}-\mathrm{C} 15-\mathrm{H} 15 \mathrm{C} \\
& \mathrm{C} 12-\mathrm{C} 16-\mathrm{H} 16 \mathrm{~A} \\
& \mathrm{C} 12-\mathrm{C} 16-\mathrm{H} 16 \mathrm{~B} \\
& \mathrm{H} 16 \mathrm{~A}-\mathrm{C} 16-\mathrm{H} 16 \mathrm{~B} \\
& \mathrm{C} 12-\mathrm{C} 16-\mathrm{H} 16 \mathrm{C} \\
& \mathrm{H} 16 \mathrm{~A}-\mathrm{C} 16-\mathrm{H} 16 \mathrm{C} \\
& \mathrm{H} 16 \mathrm{~B}-\mathrm{C} 16-\mathrm{H} 16 \mathrm{C} \\
& \text { N1-C6-C5-C7 } \\
& \text { O6-C6-C5-C12 } \\
& \text { N1-C6-C5-C12 } \\
& \text { C8-C7-C5-C4 } \\
& \text { C8-C7-C5-C6 } \\
& \text { C8-C7-C5-C12 } \\
& \text { C5-C7-C8-S9 } \\
& \text { C10-S9-C } 8-\mathrm{C} 7 \\
& \text { C4-C5-C12-C13 } \\
& \text { C6-C5-C12-C13 } \\
& \text { C7-C5-C12-C13 } \\
& \text { C4-C5-C12-C16 } \\
& \text { C6-C5-C12-C16 } \\
& \text { C7-C5-C12-C16 } \\
& \text { C16-C12-C13-C14 } \\
& \text { C5-C12-C13-C14 } \\
& \text { C12-C13-C14-C15 }
\end{aligned}
$$
108.0
109.5
109.5
109.5
109.5
109.5
109.5
109.5
109.5
109.5
109.5
109.5
109.5

$$
\begin{aligned}
& 128.90(16) \\
& 67.0(2) \\
& -111.17(17) \\
& 73.15(18) \\
& -50.13(19) \\
& -166.48(15) \\
& -173.66(12) \\
& 82.54(16) \\
& 73.5(2) \\
& -164.15(16) \\
& -46.0(2) \\
& -53.9(2) \\
& 68.47(19) \\
& -173.34(15) \\
& -60.5(2) \\
& 172.91(17)
\end{aligned}
$$$$
-171.28(19)
$$

Hydrogen-bond geometry $\left(A,{ }^{o}\right)$

\begin{tabular}{lllll}
\hline$D-\mathrm{H} \cdots A$ & $D-\mathrm{H}$ & $\mathrm{H} \cdots A$ & $D \cdots A$ & $D-\mathrm{H} \cdots A$ \\
\hline $\mathrm{N} 1-\mathrm{H} 1 \cdots \mathrm{O} 6^{\mathrm{i}}$ & $0.86(2)$ & $2.07(2)$ & $2.921(2)$ & $170(2)$ \\
$\mathrm{N} 3-\mathrm{H} 3 \cdots \mathrm{O} 4^{\mathrm{ii}}$ & $0.86(2)$ & $2.14(2)$ & $2.963(2)$ & $160(2)$ \\
\hline
\end{tabular}

Symmetry codes: (i) $-x,-y+1,-z+1$; (ii) $-x, y,-z+1 / 2$. 